\section{Evaluation of Telomerase Reverse Transcriptase Expression in Squamous Cell Carcinoma of the Skin \\ Nimita Kant ${ }^{1}$, Perumal Jayaraj ${ }^{2}$ \\ 'Department of Zoology, University of Delhi, Shivaji College, New Delhi, Delhi, India \\ ${ }^{2}$ Department of Zoology, University of Delhi, Sri Venkateswara College, New Delhi, Delhi, India}

Address for correspondence: Nimita Kant, MSc, Department of Zoology, University of Delhi, Shivaji College, RIng Road, Raja Garden, New Delhi 110027, India (e-mail: nimita@shivaji.du.ac.in).

\section{Abstract}

Introduction Squamous cell carcinoma (SCC) is highly invasive malignant tumor showing keratinocytic differentiation and is often associated with chronic exposure to UV light. Telomerase is RNA dependent DNA polymerase that causes addition of telomeric repeat DNA sequences to chromosomal ends. Recently, UV signature mutations have been identified in core promoter region of TERT gene, which encodes the main catalytic subunit leading to overexpression in cutaneous melanoma. However, its role and expression pattern have not been studied in eyelid skin SCC.

Objectives Present study aimed to analyze the presence of telomerase reverse transcriptase (TERT) in eyelid SCC, as its expression pattern and mutational status have not been studied in SCC.

Materials and Methods Nineteen cases of eyelid SCC were evaluated for the presence of TERT protein using monoclonal antibody against TERT, and its mutational status was verified using PCR and DNA sequencing. Bioedit software was used for analyzes and primers were vindicated using NCBI Primer Blast. Results were correlated with clinicopathological features of SCC.

Results A C to T mutation was observed in 6 of 19 SCC cases. Positive expression of TERT was found in $57 \%$ of the cases analyzed and it showed a significant association with keratinocytic differentiation $(p=0.04)$.

Conclusion Relation between TERT promoter mutation and TERT immunohistochemistry is studied for the first time in eyelid skin SCC. Our results suggested that overexpression of TERT may contribute to the aggressive behavior associated with SCC and such patients may warrant aggressive treatment.

Keywords: squamous cell carcinoma, telomerase reverse transcriptase, eyelid, promoter mutation, immunohistochemistry

\section{An Analysis of Acute Adverse Drug Reactions Occurring in Day Care Chemotherapy Setting in a Tertiary Care Cancer Centre}

Senthil J. R. ${ }^{1}$, Santa A. ${ }^{1}$, Pavan KB ${ }^{1}$, Rakesh P. ${ }^{1}$, Pravanika G, Sekhar ${ }^{1}$, Narendra $\mathrm{Ch}^{1}$, Krishna MMVT ${ }^{1}$

'Basavatharakam Indo American Cancer Hospital and Research Institute, Hyderabad, Telangana, 500073, India

Address for correspondence: Krishna Mohan, MVT, DNB(Med), DM, MNAMS, PDCR, Medical Oncology, Basavatharakam Indo American Cancer Hospital and Research Institute, Hyderabad, Telangana, India (e-mail: krishna.mvt@gmail.com).

\section{Abstract}

Introduction Acute adverse drug reactions (ADRs) in day care chemotherapy are not uncommon and easily manageable many a time. However, sometimes they may lead to untoward events. It is of paramount importance to document and analyze such events in contemporary medical oncology practice for the best utilization and planning of available personnel and resources.

Objectives This study was aimed to analyze the acute ADRs occurring in day care cancer chemotherapy setting.

Materials and Methods All acute ADRs reported in day care cancer chemotherapy setting, during the administration of chemotherapy, at Basavatarakam Indo American Cancer Hospital, Hyderabad, Telangana, India, were included in the study from June 15, 2020 to September 30, 2020. The ADRs were classified in to anaphylactic, allergic, and gastrointestinal (nausea/vomiting/heart burns/chest tightness). All ADRs were graded according to CTCAE version 5.0. Suspected drugs, time to reaction, and corrective measures were analyzed.

Results During the study period, a total of 8,600 sessions of day care chemotherapy were administered. ADRs were noticed in 83 cases $(\sim 1 \%)$. Among the reported ADRs, anaphylactic reactions were noted in 20 patients (24\%); allergic reactions of grades 1 and 2 were noted in 41 patients (49\%). Gastrointestinal ADRs were noted in 30 patients (36\%). Adverse reactions are mostly seen in oxaliplatin $(22.8 \%)$, rituximab (14.4\%), paclitaxel (15.6\%), carboplatin (13.2\%), and docetaxel (7.2\%). In grade-I (10\%) and grade-II (63\%) resections, supportive treatment was provided and chemotherapy was continued. Grade-III ADRs were noted in 21 patients (25\%) out of whom, 3 patients required short-term intensive care, chemotherapy was withheld until the next cycle in one patient, and chemotherapy regimen was changed in 3 patients. No patient died of ADR.

Conclusion Serious ADRs are rare in contemporary medical oncology practice during day care chemotherapy administration. Most acute ADRs were easily managed.

Keywords: ADRs, chemotherapy sessions, type of reactions, severity 